\title{
Evaluating the relationship between clinical and demographic characteristics of insulin-using people with diabetes and their health outcomes: a cluster analysis application
}

Elizabeth L Eby ( $\sim$ ebye@lilly.com )

Eli Lilly (United States)

Alison Edwards

CVS Health Clinical Trial Services LLC

Eric Meadows

Eli Lilly (United States)

Ilya Lipkovich

Eli Lilly (United States)

Brian D Benneyworth

Eli Lilly (United States)

Kenneth Snow

CVS Health Clinical Trial Services LLC

Research Article

Keywords: Diabetes, Insulin, Healthcare claims data, Cluster analysis, Healthcare utilization

Posted Date: February 23rd, 2021

DOI: https://doi.org/10.21203/rs.3.rs-199556/v1

License: (c) (1) This work is licensed under a Creative Commons Attribution 4.0 International License. Read Full License

Version of Record: A version of this preprint was published at BMC Health Services Research on July 8th, 2021. See the published version at https://doi.org/10.1186/s12913-021-06603-0. 


\section{Abstract}

Background: The aim of this study was to determine how clusters or subgroups of insulin-treated people with diabetes, based upon healthcare resource utilization, select social demographic and clinical characteristics, and diabetes management parameters, are related to health outcomes including acute care visits and hospital admissions.

Methods: This was a non-experimental, retrospective cluster analysis. We utilized Aetna administrative claims data to identify insulin-using people with diabetes with service dates from 01 January 2015 to 30 June 2018. The study included adults over the age of 18 years who had a diagnosis of type 1 (T1DM) or type 2 diabetes mellitus (T2DM) on insulin therapy and had Aetna medical and pharmacy coverage for at least 18 months (6 months prior and 12 months after their index date, defined as either their first insulin prescription fill date or their earliest date allowing for 6 months' prior coverage). We used K-means clustering methods to identify relevant subgroups of people with diabetes based on 13 primary outcome variables.

Results: A total of 100,650 insulin-using people with diabetes were identified in the Aetna administrative claims database and met study criteria, including 11,826 (11.7\%) with T1DM and 88,824 (88.3\%) with T2DM. Of these 79,053 (78.5\%) people were existing insulin users. Seven distinct clusters were identified with different characteristics and potential risks of diabetes complications. Overall, clusters were significantly associated with differences in healthcare utilization (emergency room visits, inpatient admissions, and total inpatient days) after multivariable adjustment.

Conclusions: This novel analysis of healthcare claims data using clustering methodologies identified meaningful subgroups of patients with diabetes using insulin. The subgroups differed in comorbidity burden, healthcare utilization, and demographic factors which could be used to identify higher risk patients and/or guide the management and treatment of diabetes.

\section{Introduction/background}

Diabetes is a complex, chronic illness that affects about 30 million people or $9.4 \%$ of the population in the United States [1, 2]. The population with diabetes continues to grow and the percentage of adults with diabetes increases with age, where approximately $25 \%$ of all adults over 65 years of age have diabetes. [3]. Diabetes remains the seventh leading cause of death in the United States with approximately 80,000 death certificates in 2015 listing diabetes as the underlying cause of death [3]. Although the actual number of diabetes-associated deaths may be higher as diabetes is often underreported as a cause of death [3].

The majority of people with diabetes are classified as having type 2 diabetes mellitus (T2DM), occurring in about 90-95\% of diagnosed cases [4,5], with approximately $5 \%$ of diagnosed cases classified as type 1 diabetes mellitus (T1DM) [4]. T1DM is characterized by the lack of insulin production, and as such patients require insulin to survive [5]. T2DM is characterized by the body's resistance to insulin action and is usually diagnosed in adults. Genetic factors and lifestyle play an important role in disease progression for people with either T1DM or T2DM [4].

People with diabetes visit physician offices and emergency rooms (ER) more frequently than people without diabetes, are more likely to be admitted to the hospital, and more commonly receive home health care [6]. The financial burden of diabetes to individuals and society is estimated to be a total of $\$ 327$ billion, including $\$ 237$ billion in direct costs and $\$ 90$ billion in reduced productivity in $2018[6,3]$. Healthcare expenditures are about 2.3 times higher for people with diabetes than people without diabetes with an average medical diabetes-related expenditures of approximately $\$ 9,600$ per year in the United States [6]

Although all people with T1DM use insulin, insulin use varies among people with T2DM. Most are initially managed with oral hypoglycemic agents but over time, most will ultimately utilize insulin. The majority of the diabetic population uses injection-based administration with insulin pens or vials and syringes while the remainder uses insulin pumps (continuous subcutaneous insulin infusion) $[7,8]$. Since 2005 , there has been a significant increase in the use of insulin pens while the use of vials and syringes to deliver insulin has decreased over time [9]. Insulin pens have simplified the administration of insulin, resulting in more accurate and easier delivery of insulin relative to vials and syringes [10]. Pumps deliver rapid-acting insulin throughout the day. Diabetesrelated technologies are available to monitor blood glucose and include the standard blood glucose monitor (single reading) and continuous blood glucose monitoring systems (real time and intermittently scanned) [7].

In 2005-2012, among patients who had any insulin use, only $31.4 \%$ had an hemoglobin $\mathrm{A} 1 \mathrm{c}$ (HbA1c) $<7 \%$; therefore, as glycemic control is still not attained by most people with diabetes, there is a need for new approaches to identify subgroups of people with diabetes (T1DM or T2DM) who might have risk factors that could impact treatment decisions and targeted disease management [11]. Given the differences across the US population especially in disease severity and utilization of healthcare, we sought insight from a large, real-world database. In this study, we used clustering techniques to identify subgroups of people treated with insulin based upon healthcare resource utilization, select social demographic and clinical characteristics, and diabetes management parameters. We then related these subgroups to health outcomes, including ER visits, hospital admissions, and total inpatient days.

\section{Methods}

\section{Study design and data sources}

We used a non-experimental, retrospective design in this study utilizing Aetna's administrative claims data containing membership, eligibility, medical claims, pharmacy claims, laboratory test results, and data derived for Aetna's care management processes (Health Profile Database [12]) for Aetna fully insured Commercial and Medicare Advantage members, with services dates from 01 January 2015 to 30 June 2018. All of the data used in this study were fully deidentified and Health Insurance Portability and Accountability Act compliant. 


\section{Sample selection and patient population}

Using the International Classification of Diseases Ninth and Tenth Revision (ICD9-CM v1 and ICD10CM) diagnostic codes, the study included adults 18 years of age and older who had a diagnosis of T1DM or T2DM who utilized insulin therapy and had Aetna medical and pharmacy coverage for at least 18 continuous months ( 6 months of coverage prior and 12 months after their index date, defined as either their first insulin prescription fill date or their earliest insulin fill date allowing for 6 months prior coverage; Fig. 1). People with diabetes were excluded from the analyses if any of the following criteria were met during the entire study period: had $\geq 1$ inpatient or outpatient medical claim with a diagnosis in any position of gestational diabetes, steroid-induced diabetes, or metastatic cancer; had indications of hospice use; or were enrolled in Aetna's Compassionate Care Program.

The study was approved by the Sterling Institutional Review Board (Atlanta, Georgia, USA).

\section{Statistical analysis}

Descriptive analyses were performed on the overall study population and stratified by T1DM and T2DM, as well as by cluster assignment.

Descriptive statistics were generated for all demographic characteristics and utilization measures as applicable to the type of variable. Continuous variables were described using means with standard deviations (SD) or medians with first and third quartiles if data were highly skewed. Categorical and binary variables were described using counts and percentages. Healthcare utilization were reported as means and SD and number of patients with $\geq 1$ visit.

\section{Cluster analysis}

A cluster analysis was performed on pre-period variables that were hypothesized to be both related to outcomes and to the use of diabetes technology (blood glucose monitor [BGM], continuous glucose monitoring [CGM], or insulin pumps). They included age, number of endocrinologist visits, diabetes complications severity index (DCSI) [13], Charlson comorbidity index (CCI) [14], number of HbA1c tests, number of months on insulin, number of ER visits, total number of inpatient days, number of discordant comorbidities, number of concordant comorbidities, number of medical claims, proportion diabetesrelated claims, and estimated income. Concordant comorbidities were defined as those that share the same pathophysiologic risk profile and management plan as with diabetes, whereas discordant conditions are those that are not directly related to diabetes in either their pathophysiology or management [15]. As the underlying pathophysiology differs for T1DM and T2DM, concordant and discordant were defined separately for each type of diabetes (see Supplemental Materials Table S1). Clinically dominant comorbidities, those that are so complex or serious that they tend to eclipse the management of other conditions [15], were considered but not found in the study population. Claims were considered diabetes related if they contained a diagnosis for diabetes in any position. All 13 cluster variables had significant bivariate associations with utilization of diabetes technology and were standardized according to the type of variable. We opted to use variables associated with the utilization of diabetes technology in the cluster analysis to identify unexpected patterns in the characteristics of patients who utilized different types of technology. After clustering, utilization of diabetes technology along with other relevant variables such as diabetes type, insurer, and HbA1c levels (when available) were reported to help characterize the clusters.

We utilized K-means methodology to identify the clusters based on the 13 variables mentioned above. To determine the optimal number of clusters, we used the Jump method [16], a non-parametric method for choosing the number of clusters based on "distortion" which is a measure of within-cluster dispersion. We chose the Jump method as it is a simple and readily available method that has performed well against other competing methods in simulated data analyses [16].

To identify which variables played a key role in the formation of clusters and to obtain simple descriptive rules for clusters, classification and regression trees (CART) were used with cluster assignment as the "outcome" variable and all variables used in clustering as the "predictors." This analysis was performed using the Ctree function in the R package partykit.

Outcomes of interest, measured in the 12-month follow-up period, included: all-cause ER utilization, allcause inpatient hospitalization, and total inpatient days. To determine if the clusters were associated with the outcomes measured during the study, multivariable generalized linear models were applied with clusters as the independent variables and other covariates or interactions to account for confounding. The generalized linear models included binomial distribution and logit link function (ER and inpatient hospitalization outcomes) and negative binomial distribution with log link function (total inpatient days outcome). For the multivariable regression analyses, we used a stepwise selection methodology (or backward elimination) with variable removal when $p \geq 0.05$. Akaike Information Criterion was also used to determine the best fit model. All statistical analyses were conducted using SAS Enterprise Guide Version 6.1 (SAS Institute Inc., Cary, NC, USA) or R version 3.2.5 (R Foundation for Statistical Computing, Vienna, Austria).

\section{Results}

\section{Study population}

A total of 100,650 insulin-using people with diabetes met the study criteria, including 11,826 (11.7\%) with T1DM and 88,824 (88.3\%) with T2DM. Table 1 shows the pre-period demographic characteristics for the total population and differences between people with T1DM and T2DM. The majority of study patients (78.5\%) were existing insulin users. The mean age (SD) was 62.2 (14.2) years with a mean age (SD) of 45.3 (16.3) years for those with T1DM and 64.4 (12.3) years for those with T2DM. There were slightly more males than females ( $51.6 \%$ male versus $48.3 \%$ female). The median (interquartile range) household income was $\$ 54,143(\$ 43,184$ to $\$ 69,606)$ for the total population with a slightly higher income for those with T1DM of $\$ 63,304$ (\$49,195 to $\$ 81,547)$ compared to T2DM of $\$ 53,299(\$ 42,675$ to $\$ 68,059)$. 
Table 1

Pre-period ${ }^{1}$ demographic and clinical characteristics (baseline)

\begin{tabular}{|c|c|c|c|c|c|c|c|}
\hline & \multicolumn{2}{|l|}{ Total } & \multicolumn{2}{|l|}{ T1DM } & \multicolumn{2}{|l|}{ T2DM } & \multirow[t]{2}{*}{$\mathbf{p}$} \\
\hline & $\mathrm{N}$ & $\%$ & $\mathrm{~N}$ & $\%$ & $\mathrm{~N}$ & $\%$ & \\
\hline Total Number of People With Diabetes & 100,650 & $100 \%$ & 11,826 & $11.7 \%$ & 88,824 & $88.3 \%$ & - \\
\hline Subgroup of Existing Insulin Users During the Pre-period & 79,053 & $78.5 \%$ & 10,975 & $92.8 \%$ & 68,078 & $76.6 \%$ & $\begin{array}{l}< \\
0.0001\end{array}$ \\
\hline \multicolumn{8}{|l|}{ Characteristics } \\
\hline Age $(n, \%)$ & & & & & & & $\begin{array}{l}< \\
0.0001\end{array}$ \\
\hline $18-40$ & 8,068 & $8.0 \%$ & 4,843 & $41.0 \%$ & 3,225 & $3.6 \%$ & \\
\hline $41-60$ & 32,960 & $32.7 \%$ & 4,713 & $39.9 \%$ & 28,247 & $31.8 \%$ & \\
\hline$>60$ & 59,622 & $59.2 \%$ & 2,270 & $19.2 \%$ & 57,352 & $64.6 \%$ & \\
\hline Age (mean, SD) & 62.18 & 14.24 & 45.26 & 16.27 & 64.43 & 12.30 & $\begin{array}{l}< \\
0.0001\end{array}$ \\
\hline Gender (n, \%) & & & & & & & 0.0003 \\
\hline Male & 51,983 & $51.6 \%$ & 6,290 & $53.2 \%$ & 45,693 & $51.4 \%$ & \\
\hline Female & 48,651 & $48.3 \%$ & 5,532 & $46.8 \%$ & 43,119 & $48.5 \%$ & \\
\hline Missing & 16 & $0.0 \%$ & 4 & $0.0 \%$ & 12 & $0.0 \%$ & \\
\hline Business Line $(\mathrm{n}, \%)$ & & & & & & & $\begin{array}{l}< \\
0.0001\end{array}$ \\
\hline Commercial & 43,573 & $43.3 \%$ & 9,566 & $80.9 \%$ & 34,007 & $38.3 \%$ & \\
\hline Medicare advantage & 57,077 & $56.7 \%$ & 2,260 & $19.1 \%$ & 54,817 & $61.7 \%$ & \\
\hline \multicolumn{8}{|l|}{ Household Income, in US Dollars } \\
\hline $\mathrm{n}(\%)$ & 100,196 & $99.5 \%$ & 11,801 & $99.8 \%$ & 88,395 & $99.5 \%$ & \\
\hline Median, Q1 - Q3 & $\$ 54,143$ & $\begin{array}{l}\$ 43,184- \\
\$ 69,606\end{array}$ & $\$ 63,304$ & $\begin{array}{l}\$ 49,195- \\
\$ 81,547\end{array}$ & $\$ 53,299$ & $\begin{array}{l}\$ 42,675- \\
\$ 68,059\end{array}$ & $\begin{array}{l}<.0001 \\
0 .\end{array}$ \\
\hline \multicolumn{8}{|l|}{ Medical Claims } \\
\hline Number of Medical Claims (median, Q1 - Q3) & 7.00 & $4.00-15.00$ & 5.00 & $3.00-10.00$ & 8.00 & $4.00-15.00$ & $<.0001$ \\
\hline $\begin{array}{l}\text { Proportion of Medical Claims }{ }^{2,3} \text { That are Diabetes Related } \\
\text { (mean, SD) }\end{array}$ & 0.54 & 0.31 & 0.60 & 0.33 & 0.52 & 0.30 & $<.0001$ \\
\hline \multicolumn{8}{|l|}{ Number of HbA1c Tests } \\
\hline $\begin{array}{l}\text { One or More Tests Performed } \\
(\mathrm{n}, \%)\end{array}$ & 60,668 & $60.3 \%$ & 7,137 & $60.4 \%$ & 53,531 & $60.3 \%$ & 0.86 \\
\hline Mean, SD & 0.91 & 0.93 & 0.89 & 0.88 & 0.91 & 0.94 & 0.0007 \\
\hline \multicolumn{8}{|l|}{ Latest HbA1c Value } \\
\hline $\mathrm{n}(\%)$ & 31,410 & $31.2 \%$ & 3,417 & $28.9 \%$ & 27,993 & $31.5 \%$ & \\
\hline Mean, SD & 8.84 & 1.94 & 8.38 & 1.64 & 8.89 & 1.96 & $\dot{0} 0001$ \\
\hline Insulin Administration and Diabetes Technology (n, \%) & & & & & & & $\dot{0} 0001$ \\
\hline CGM with pump & 1,974 & $2.0 \%$ & 1,734 & $14.7 \%$ & 240 & $0.3 \%$ & \\
\hline
\end{tabular}

Abbreviations: $\mathrm{BGM}=$ blood glucose monitoring; $\mathrm{CGM}=$ continuous glucose monitoring; $\mathrm{HbA} 1 \mathrm{c}=$ hemoglobin $\mathrm{A} 1 \mathrm{c} ; \mathrm{n}, \mathrm{N}=$ number of people with diabetes; Q1 = first quartile; Q3 = third quartile; SD = standard deviation; T1DM = type 1 diabetes mellitus; T2DM = type 2 diabetes mellitus; US = United States.

${ }^{1}$ Pre-period was defined as 6 months prior to index date

2 Proportion is set to zero for those members who had zero medical claims overall during the baseline period.

${ }^{3}$ Diabetes related determined by diagnosis in any diagnostic position on a medical claim. 


\begin{tabular}{|c|c|c|c|c|c|c|c|}
\hline & Total & & T1DM & & T2DM & & $\mathbf{p}$ \\
\hline CGM with pen & 800 & $0.8 \%$ & 342 & $2.9 \%$ & 458 & $0.5 \%$ & \\
\hline CGM with vial & 277 & $0.3 \%$ & 200 & $1.7 \%$ & 77 & $0.1 \%$ & \\
\hline BGM with pump & 2,098 & $2.1 \%$ & 1,516 & $12.8 \%$ & 582 & $0.7 \%$ & \\
\hline BGM with pen & 23,078 & $22.9 \%$ & 2,032 & $17.2 \%$ & 21,046 & $23.7 \%$ & \\
\hline BGM with vial & 8,380 & $8.3 \%$ & 1,078 & $9.1 \%$ & 7,302 & $8.2 \%$ & \\
\hline No CGM or BGM/pump & 2,410 & $2.4 \%$ & 1,236 & $10.5 \%$ & 1,174 & $1.3 \%$ & \\
\hline No CGM or BGM/pen & 44,108 & $43.8 \%$ & 2,208 & $18.7 \%$ & 41,900 & $47.2 \%$ & \\
\hline No CGM or BGM/vial & 17,525 & $17.4 \%$ & 1,480 & $12.5 \%$ & 16,045 & $18.1 \%$ & \\
\hline Type of Insulin (n, \%) & & & & & & & $<.0001$ \\
\hline $\mathrm{N}$ & 77,912 & $77.4 \%$ & 10,524 & $89.0 \%$ & 67,388 & $75.9 \%$ & \\
\hline Basal only & 31,917 & $41.0 \%$ & 893 & $8.5 \%$ & 31,024 & $46.0 \%$ & \\
\hline Bolus only & 8,949 & $11.5 \%$ & 4,742 & $45.1 \%$ & 4,207 & $6.2 \%$ & \\
\hline Both & 37,046 & $47.5 \%$ & 4,889 & $46.5 \%$ & 32,157 & $47.7 \%$ & \\
\hline Months on Insulin (mean, SD) & 3.99 & 3.48 & 4.81 & 3.26 & 3.89 & 3.49 & $<.0001$ \\
\hline \multicolumn{8}{|l|}{ Clinical Characteristics } \\
\hline Number of Concordant Comorbidities (mean, SD) & 4.20 & 2.57 & 0.87 & 1.17 & 4.64 & 2.38 & $<.0001$ \\
\hline Number of Discordant Comorbidities (mean, SD) & 1.07 & 1.11 & 1.83 & 1.62 & 0.97 & 0.98 & $<.0001$ \\
\hline Charlson Comorbidity Index (CCI) (mean, SD) & 1.86 & 1.60 & 1.40 & 1.21 & 1.92 & 1.63 & $<.0001$ \\
\hline Diabetes Complication Severity Index (DCSI) (mean, SD) & 1.07 & 1.47 & 0.58 & 1.09 & 1.13 & 1.50 & $<.0001$ \\
\hline \multicolumn{8}{|c|}{$\begin{array}{l}\text { Abbreviations: BGM = blood glucose monitoring; } \mathrm{CGM}=\text { continuous glucose monitoring; HbA1c = hemoglobin } \mathrm{A} 1 \mathrm{c} ; \mathrm{n}, \mathrm{N}=\text { number of people with diabetes; } \\
\mathrm{Q} 1=\text { first quartile; } \mathrm{Q} 3=\text { third quartile; } \mathrm{SD}=\text { standard deviation; T1DM = type } 1 \text { diabetes mellitus; T2DM = type } 2 \text { diabetes mellitus; US = United States. }\end{array}$} \\
\hline \multicolumn{8}{|l|}{${ }^{1}$ Pre-period was defined as 6 months prior to index date } \\
\hline \multicolumn{8}{|c|}{2 Proportion is set to zero for those members who had zero medical claims overall during the baseline period. } \\
\hline
\end{tabular}

Overall, the mean (SD) CCI score was 1.9 (1.6) and the mean DCSI was 1.1 (1.5), both of which were lower for those with T1DM (Table 1). A total of 60,668 people $(60.3 \%)$ had one or more $\mathrm{HbA} 1 \mathrm{c}$ test performed and the average $\mathrm{HbA} 1 \mathrm{c}$ value was $8.8 \%$ among those individuals (31.2\%) with values in the database. Approximately $6.4 \%$ of the population used an insulin pump (37.9\% T1DM and 2.2\% T2DM) and 67.5\% used a pen (38.7\% T1DM and 71.4\% T2DM). Blood glucose meters were utilized by approximately one-third of the population (33.3\%). On the other hand, CGMs were only used by $3.0 \%$ overall, where T1DM patients had greater use compared to those with T2DM $(19.2 \%$ and $<1 \%$, respectively).

The healthcare utilization over the 6-month pre-period is shown in Table 2. The majority of the population had primary care physician (PCP) visits (72.3\%), but the proportion with endocrinologist and cardiologist visits was lower at $21.3 \%$ and $17.8 \%$, respectively. Inpatient hospitalizations occurred for $14.0 \%$ of the population and $18.8 \%$ had ER visits. Overall, people with T1DM had lower utilization of all-cause and diabetes-related healthcare services than people with T2DM, with the exception of endocrinologist visits. 
Table 2

Pre-period ${ }^{1}$ healthcare utilization (baseline)

\begin{tabular}{|c|c|c|c|c|c|c|c|}
\hline & \multirow{2}{*}{$\begin{array}{l}\text { Total } \\
\mathrm{N}\end{array}$} & \multicolumn{2}{|c|}{ T1DM } & \multicolumn{2}{|c|}{ T2DM } & \multicolumn{2}{|c|}{$\mathbf{p}$} \\
\hline & & $\%$ & $\mathrm{~N}$ & $\%$ & $\mathrm{~N}$ & $\%$ & \\
\hline Total Number of People With Diabetes & 100,650 & $100 \%$ & 11,826 & $11.7 \%$ & 88,824 & $88.3 \%$ & - \\
\hline \multicolumn{8}{|l|}{ Healthcare Resource Utilization ${ }^{2}$} \\
\hline \multicolumn{8}{|l|}{ All-cause Utilization } \\
\hline All-cause ER visits $(n>0, \%)$ & 18,930 & $18.8 \%$ & 1,452 & $12.3 \%$ & 17,478 & $19.7 \%$ & $<0.0001$ \\
\hline All-cause ER visits (mean, SD) & 0.30 & 0.85 & 0.18 & 0.59 & 0.32 & 0.88 & $<0.0001$ \\
\hline All-cause IP visits ( $\mathrm{n}>0, \%)$ & 14,048 & $14.0 \%$ & 761 & $6.4 \%$ & 13,287 & $15.0 \%$ & $<0.0001$ \\
\hline All-cause IP visits (mean, SD) & 0.21 & 0.64 & 0.09 & 0.40 & 0.23 & 0.67 & $<0.0001$ \\
\hline All-cause IP days (mean, SD) & 1.74 & 8.24 & 0.44 & 3.45 & 1.92 & 8.67 & $<0.0001$ \\
\hline All-cause IP days for patients with $\geq 1$ admit (mean, SD) & 12.48 & 18.79 & 6.79 & 11.94 & 12.80 & 19.05 & $<0.0001$ \\
\hline All-cause PCP visits $(n>0, \%)$ & 72,779 & $72.3 \%$ & 6,569 & $55.5 \%$ & 66,210 & $74.5 \%$ & $<0.0001$ \\
\hline All-cause PCP visits (mean, SD) & 2.05 & 2.27 & 1.20 & 1.66 & 2.16 & 2.32 & $<0.0001$ \\
\hline All-cause Endocrinologist visits $(n>0, \%)$ & 21,436 & $21.3 \%$ & 5,055 & $42.7 \%$ & 16,381 & $18.4 \%$ & $<0.0001$ \\
\hline All-cause Endocrinologist visits (mean, SD) & 0.36 & 0.80 & 0.69 & 0.97 & 0.31 & 0.76 & $<0.0001$ \\
\hline All-cause Cardiologist visits ( $n>0, \%)$ & 17,954 & $17.8 \%$ & 884 & $7.5 \%$ & 17,070 & $19.2 \%$ & $<0.0001$ \\
\hline All-cause Cardiologist visits (mean, SD) & 0.30 & 0.83 & 0.11 & 0.50 & 0.32 & 0.87 & $<0.0001$ \\
\hline No visit to PCP, Endocrinologist, or Cardiologist (n, \%) & 18,671 & $18.6 \%$ & 2,677 & $22.6 \%$ & 15,994 & $18.0 \%$ & $<0.0001$ \\
\hline \multicolumn{8}{|l|}{ Diabetes-related Utilization ${ }^{3}$} \\
\hline Diabetes-related ER visits $(n>0, \%)$ & 15,057 & $15.0 \%$ & 1,163 & $9.8 \%$ & 13,894 & $15.6 \%$ & $<0.0001$ \\
\hline Diabetes-related ER visits (mean, SD) & 0.23 & 0.71 & 0.14 & 0.51 & 0.24 & 0.73 & $<0.0001$ \\
\hline Diabetes-related IP visits $(n>0, \%)$ & 13,536 & $13.4 \%$ & 750 & $6.3 \%$ & 12,786 & $14.4 \%$ & $<0.0001$ \\
\hline Diabetes-related IP visits (mean, SD) & 0.20 & 0.60 & 0.08 & 0.39 & 0.21 & 0.62 & $<0.0001$ \\
\hline Diabetes-related IP days (mean, SD) & 1.59 & 7.58 & 0.40 & 3.15 & 1.74 & 7.98 & $<0.0001$ \\
\hline Diabetes-related IP days for patients with $\geq 1$ admit (mean, SD) & 11.79 & 17.53 & 6.37 & 10.90 & 12.11 & 17.79 & $<0.0001$ \\
\hline \multicolumn{8}{|c|}{$\begin{array}{l}\text { Abbreviations: } \mathrm{ER}=\text { emergency room; HbA1c = hemoglobin A1c; IP = inpatient; } \mathrm{n}, \mathrm{N}=\text { number of people with diabetes; } \mathrm{PCP}=\text { primary care physician; } \mathrm{Q} 1= \\
\text { first quartile; } \mathrm{Q} 3=\text { third quartile; } \mathrm{SD}=\text { standard deviation; } \mathrm{T} 1 \mathrm{DM}=\text { type } 1 \text { diabetes mellitus; } \mathrm{T} 2 \mathrm{DM}=\text { type } 2 \text { diabetes mellitus }\end{array}$} \\
\hline \multicolumn{8}{|l|}{${ }^{1}$ Pre-period was defined as 6 months prior to index date } \\
\hline \multicolumn{8}{|c|}{${ }^{2}$ Statistical comparisons for average utilization were conducted using Wilcoxon Rank Sum tests. } \\
\hline
\end{tabular}

\section{Cluster formation and analysis}

We identified seven clusters of people with diabetes, which had distinguishable characteristics and risk factors. Characteristics of the seven identified clusters are shown in Table 3. Of the 13 clustering variables studied using the CART analysis, number of endocrinology visits, total inpatient days, concordant comorbidities, number of ER visits, comorbidity burden as measured by CCI and DCSI scores, and percentage of diabetes-related medical claims were the most important factors for cluster formation. Figure 2 illustrates the CART analysis after the clusters were formed. 
Table 3

Description of people with diabetes identified through cluster analysis

\begin{tabular}{|c|c|c|c|c|c|c|c|}
\hline & \multicolumn{7}{|l|}{ CLUSTER NUMBER } \\
\hline & 1 & 2 & 3 & 4 & 5 & 6 & 7 \\
\hline Cluster Label & $\begin{array}{l}\text { Low ER Utilizers } \\
\text { with High } \\
\text { Comorbid Burden }\end{array}$ & $\begin{array}{l}\text { Endocrinology } \\
\text { Utilizers with } \\
\text { Concordant } \\
\text { Comorbidities }\end{array}$ & $\begin{array}{l}\text { Low ER Utilizers } \\
\text { with High } \\
\text { Diabetes-related } \\
\text { Claims without } \\
\text { Discordant } \\
\text { Comorbidities }\end{array}$ & $\begin{array}{l}\text { High Inpatient } \\
\text { Utilizers with High } \\
\text { Comorbid Burden }\end{array}$ & High ER Utilizers & $\begin{array}{l}\text { Endocrinology } \\
\text { Utilizers without } \\
\text { Concordant } \\
\text { Comorbidities }\end{array}$ & $\begin{array}{l}\text { Lowes } \\
\text { Utilizer }\end{array}$ \\
\hline Number of & 22,508 & 13,986 & 22,880 & 5,629 & 11,351 & 10,049 & 14,247 \\
\hline$(n, \%)$ & $(22.4 \%)$ & $(13.9 \%)$ & $(22.7 \%)$ & $(5.6 \%)$ & $(11.3 \%)$ & $(10.0 \%)$ & (14.2\% \\
\hline Age (mean, SD) & 69.80 & 65.16 & 60.71 & 69.54 & 64.92 & 38.34 & 61.31 \\
\hline (years) & $(9.85)$ & $(10.83)$ & $(10.83)$ & (11.98) & $(12.54)$ & (12.16) & $(12.51)$ \\
\hline Male (\%) & $48.0 \%$ & $50.8 \%$ & $45.4 \%$ & $48.9 \%$ & $54.8 \%$ & $43.0 \%$ & $49.5 \%$ \\
\hline $\begin{array}{l}\text { Household } \\
\text { Income (US } \\
\text { dollars) }\end{array}$ & $\begin{array}{l}\$ 51,665 \\
(\$ 42,344-\$ 65,516)\end{array}$ & $\begin{array}{l}\$ 59,733 \\
(\$ 47,149-\$ 77,449)\end{array}$ & $\begin{array}{l}\$ 51,879 \\
(\$ 42,139-\$ 66,124)\end{array}$ & $\begin{array}{l}\$ 53,612 \\
(\$ 43,017-\$ 68,301)\end{array}$ & $\begin{array}{l}\$ 49,154 \\
(\$ 40,163-\$ 61,588)\end{array}$ & $\begin{array}{l}\$ 65,972 \\
(\$ 51,625-\$ 84,930)\end{array}$ & $\begin{array}{l}\$ 54,10 \\
(\$ 43,1 !\end{array}$ \\
\hline \multicolumn{8}{|l|}{$\begin{array}{l}\text { Median (Q1 - } \\
\text { Q3) }\end{array}$} \\
\hline $\begin{array}{l}\text { Medicare } \\
\text { Advantage (\%) }\end{array}$ & $80.3 \%$ & $62.2 \%$ & $42.6 \%$ & $81.5 \%$ & $72.5 \%$ & $3.8 \%$ & $51.7 \%$ \\
\hline Diabetes Type & $3.3 \%$ & $13.6 \%$ & $2.2 \%$ & $2.1 \%$ & $3.6 \%$ & $69.6 \%$ & $8.1 \%$ \\
\hline \multicolumn{8}{|l|}{ T1DM (\%) } \\
\hline \multicolumn{8}{|l|}{$\begin{array}{l}\text { Number of } \\
\mathrm{HbA1c} \text { Tests }\end{array}$} \\
\hline $\begin{array}{l}\text { One or more } \\
\text { HbA1c tests } \\
\text { performed (\%) }\end{array}$ & $66.4 \%$ & $75.8 \%$ & $71.3 \%$ & $54.3 \%$ & $63.8 \%$ & $63.0 \%$ & $15.2 \%$ \\
\hline Mean (SD) & $1.01(0.91)$ & $1.29(1.00)$ & $1.02(0.83)$ & $0.89(1.21)$ & $1.00(0.99)$ & $0.87(0.81)$ & $0.17(0$ \\
\hline \multicolumn{8}{|l|}{$\begin{array}{l}\text { Latest HbA1c } \\
\text { Value }\end{array}$} \\
\hline $\mathrm{N}$ & 7,007 & 6,215 & 7,494 & 1,471 & 3,260 & 3,045 & 2,918 \\
\hline Mean (SD) & $8.56(1.79)$ & $8.60(1.69)$ & $9.35(2.07)$ & $8.25(1.81)$ & $8.95(2.05)$ & $8.82(2.02)$ & $8.87(1$ \\
\hline \multicolumn{8}{|l|}{$\begin{array}{l}\text { Utilization of } \\
\text { Diabetes } \\
\text { Technology }\end{array}$} \\
\hline Pump (\%) & $2.8 \%$ & $10.6 \%$ & $2.1 \%$ & $4.2 \%$ & $4.3 \%$ & $29.2 \%$ & $1.7 \%$ \\
\hline BGM (\%) & $33.3 \%$ & $38.6 \%$ & $31.8 \%$ & $29.6 \%$ & $35.3 \%$ & $39.6 \%$ & $26.2 \%$ \\
\hline CGM (\%) & $1.0 \%$ & $6.8 \%$ & $0.8 \%$ & $1.0 \%$ & $1.2 \%$ & $14.5 \%$ & $0.3 \%$ \\
\hline \multicolumn{8}{|l|}{$\begin{array}{l}\text { Type of Insulin } \\
(\%)\end{array}$} \\
\hline Basal only & $45.0 \%$ & $27.9 \%$ & $55.3 \%$ & $32.5 \%$ & $40.8 \%$ & $17.3 \%$ & $47.4 \%$ \\
\hline Bolus only & $6.2 \%$ & $15.6 \%$ & $6.0 \%$ & $10.9 \%$ & $8.2 \%$ & $36.7 \%$ & $7.2 \%$ \\
\hline Both & $48.7 \%$ & $56.5 \%$ & $38.8 \%$ & $56.6 \%$ & $51.0 \%$ & $46.0 \%$ & $45.3 \%$ \\
\hline
\end{tabular}

Abbreviations: $\mathrm{BGM}=$ blood glucose monitor; $\mathrm{CCI}=$ Charlson Comorbidity Index; $\mathrm{CGM}=$ continuous glucose monitor; $\mathrm{DCSI}=$ diabetes complications severity ir emergency room; $\mathrm{HbA} 1 \mathrm{c}=$ hemoglobin $\mathrm{A} 1 \mathrm{c} ; \mathrm{IP}=$ inpatient; $\mathrm{n}, \mathrm{N}=$ number of people with diabetes; $\mathrm{PCP}=$ primary care physician; Q1 = first quartile; $\mathrm{Q} 3=$ third qi = standard deviation; T1DM = type 1 diabetes mellitus.

${ }^{1}$ Missing values for median household income were imputed for 454 patients for the purpose of cluster analysis.

${ }^{2}$ Proportion is set to zero for those members who had zero medical claims overall during the baseline period.

${ }^{3}$ Diabetes related determined by diagnosis in any diagnostic position on a medical claim.

${ }^{4}$ Statistical comparisons for average utilization were conducted using Kruskal-Wallis tests. 


\begin{tabular}{|c|c|c|c|c|c|c|c|}
\hline & \multicolumn{7}{|c|}{ CLUSTER NUMBER } \\
\hline $\begin{array}{l}\text { Months on } \\
\text { Insulin (mean, } \\
\text { SD) }\end{array}$ & $4.45(3.58)$ & $5.02(3.72)$ & 3.34 (3.15) & $2.68(3.18)$ & $3.70(3.47)$ & $4.29(3.25)$ & $3.87(3$ \\
\hline \multicolumn{8}{|l|}{$\begin{array}{l}\text { Clinical } \\
\text { Characteristics }\end{array}$} \\
\hline $\begin{array}{l}\text { Number of } \\
\text { Concordant } \\
\text { Comorbidities } \\
\text { (mean, SD) }\end{array}$ & $5.56(2.17)$ & $4.56(2.31)$ & $3.29(1.61)$ & $6.72(2.34)$ & $5.53(2.34)$ & $0.52(0.76)$ & $3.67(2$ \\
\hline $\begin{array}{l}\text { Number of } \\
\text { Discordant } \\
\text { Comorbidities } \\
\text { (mean, SD) }\end{array}$ & $1.40(1.07)$ & $1.46(1.26)$ & $0.50(0.74)$ & $1.44(1.16)$ & $1.31(1.14)$ & $0.89(1.10)$ & $0.88(0$ \\
\hline $\begin{array}{l}\text { Charlson } \\
\text { Comorbidity } \\
\text { Index (CCI) } \\
\text { (mean, SD) }\end{array}$ & $2.70(1.44)$ & $2.47(1.50)$ & $1.15(0.78)$ & $3.92(2.03)$ & $2.47(1.61)$ & $1.09(0.74)$ & $0.30(0$ \\
\hline $\begin{array}{l}\text { Diabetes } \\
\text { Complication } \\
\text { Severity Index } \\
\text { (DCSI) (mean, } \\
\text { SD) }\end{array}$ & 1.79 (1.39) & 1.33 (1.39) & $0.26(0.58)$ & 3.48 (1.95) & $1.66(1.53)$ & $0.22(0.56)$ & $0.15(0$ \\
\hline $\begin{array}{l}\text { Median (Q1 - } \\
\text { Q3) Number of } \\
\text { Medical Claims }\end{array}$ & $\begin{array}{l}11.00(7.00- \\
18.00)\end{array}$ & $\begin{array}{l}11.00(7.00- \\
17.00)\end{array}$ & $4.00(2.00-6.00)$ & $\begin{array}{l}43.00(30.00- \\
64.00)\end{array}$ & $\begin{array}{l}14.00(9.00- \\
23.00)\end{array}$ & $4.00(2.00-7.00)$ & $3.00(0$ \\
\hline $\begin{array}{l}\text { Proportion of } \\
\text { Medical Claims } \\
2,3 \text { That are } \\
\text { Diabetes } \\
\text { Related (mean, } \\
\text { SD) }\end{array}$ & $0.46(0.24)$ & $0.53(0.24)$ & $0.78(0.23)$ & $0.53(0.25)$ & $0.51(0.24)$ & $0.69(0.30)$ & $0.19(0$ \\
\hline \multicolumn{8}{|l|}{$\begin{array}{l}\text { Healthcare } \\
\text { Resource } \\
\text { Utilization }{ }^{4}\end{array}$} \\
\hline \multicolumn{8}{|l|}{$\begin{array}{l}\text { All-cause } \\
\text { Utilization }\end{array}$} \\
\hline $\begin{array}{l}\text { All-cause ER } \\
\text { visits (\%) }\end{array}$ & $0.0 \%$ & $10.7 \%$ & $6.2 \%$ & $45.2 \%$ & $100.0 \%$ & $12.0 \%$ & $6.5 \%$ \\
\hline $\begin{array}{l}\text { All-cause ER } \\
\text { visits (mean, } \\
\text { SD) }\end{array}$ & $0.00(0.00)$ & $0.12(0.36)$ & $0.07(0.26)$ & $0.82(1.37)$ & $1.77(1.50)$ & $0.15(0.44)$ & $0.07(0$ \\
\hline $\begin{array}{l}\text { All-cause IP } \\
\text { visits (\%) }\end{array}$ & $11.6 \%$ & $7.5 \%$ & $5.4 \%$ & $99.1 \%$ & $22.2 \%$ & $6.0 \%$ & $3.2 \%$ \\
\hline $\begin{array}{l}\text { All-cause IP } \\
\text { visits (mean, } \\
\text { SD) }\end{array}$ & $0.13(0.36)$ & $0.08(0.31)$ & $0.06(0.25)$ & $2.11(1.29)$ & $0.26(0.53)$ & $0.07(0.28)$ & $0.03(0$ \\
\hline $\begin{array}{l}\text { All-cause IP } \\
\text { days for } \\
\text { patients with } \\
\geq 1 \text { admit } \\
\text { (mean, SD) }\end{array}$ & $3.84(2.04)$ & 3.88 (2.69) & $4.07(2.81)$ & $25.34(24.57)$ & $4.13(2.75)$ & $3.61(2.96)$ & $5.03(4$ \\
\hline $\begin{array}{l}\text { All-cause PCP } \\
\text { visits (\%) }\end{array}$ & $83.7 \%$ & $71.4 \%$ & $79.8 \%$ & $81.3 \%$ & $84.1 \%$ & $55.8 \%$ & $41.8 \%$ \\
\hline
\end{tabular}

Abbreviations: $\mathrm{BGM}=$ blood glucose monitor; $\mathrm{CCI}=$ Charlson Comorbidity Index; $\mathrm{CGM}=$ continuous glucose monitor; $\mathrm{DCSI}=$ diabetes complications severity ir emergency room; HbA1c = hemoglobin A1c; IP = inpatient; $n, N=$ number of people with diabetes; PCP = primary care physician; Q1 = first quartile; $\mathrm{Q} 3=$ third qi = standard deviation; T1DM = type 1 diabetes mellitus.

${ }^{1}$ Missing values for median household income were imputed for 454 patients for the purpose of cluster analysis.

2 Proportion is set to zero for those members who had zero medical claims overall during the baseline period.

${ }^{3}$ Diabetes related determined by diagnosis in any diagnostic position on a medical claim.

${ }^{4}$ Statistical comparisons for average utilization were conducted using Kruskal-Wallis tests. 


\section{CLUSTER NUMBER}

\begin{tabular}{|c|c|c|c|c|c|c|c|}
\hline $\begin{array}{l}\text { All-cause PCP } \\
\text { visits (mean, } \\
\text { SD) }\end{array}$ & $2.60(2.32)$ & $1.73(1.88)$ & $1.87(1.58)$ & 3.44 (3.69) & 3.31 (2.97) & $1.10(1.41)$ & $0.86(1$ \\
\hline $\begin{array}{l}\text { All-cause } \\
\text { Endocrinologist } \\
\text { visits (\%) }\end{array}$ & $0.7 \%$ & $100.0 \%$ & $4.0 \%$ & $13.5 \%$ & $12.1 \%$ & $39.2 \%$ & $2.1 \%$ \\
\hline $\begin{array}{l}\text { All-cause } \\
\text { Endocrinologist } \\
\text { visits (mean, } \\
\text { SD) }\end{array}$ & $0.01(0.08)$ & $1.83(0.94)$ & $0.04(0.23)$ & $0.22(0.69)$ & $0.16(0.47)$ & $0.58(0.85)$ & $0.02(0$ \\
\hline $\begin{array}{l}\text { All-cause } \\
\text { Cardiologist } \\
\text { visits (\%) }\end{array}$ & $29.0 \%$ & $27.5 \%$ & $5.1 \%$ & $36.8 \%$ & $28.8 \%$ & $2.0 \%$ & $6.1 \%$ \\
\hline $\begin{array}{l}\text { All-cause } \\
\text { Cardiologist } \\
\text { visits (mean, } \\
\text { SD) }\end{array}$ & $0.46(0.98)$ & $0.43(0.90)$ & $0.07(0.33)$ & $0.79(1.48)$ & $0.53(1.13)$ & $0.03(0.24)$ & $0.08(0$ \\
\hline $\begin{array}{l}\text { No visit to PCP, } \\
\text { Endocrinologist } \\
\text { or Cardiologist } \\
(\%)\end{array}$ & $12.4 \%$ & $0.0 \%$ & $17.4 \%$ & $12.7 \%$ & $11.2 \%$ & $21.4 \%$ & $54.6 \%$ \\
\hline \multicolumn{8}{|l|}{$\begin{array}{l}\text { Diabetes- } \\
\text { related } \\
\text { Utilization }^{3}\end{array}$} \\
\hline $\begin{array}{l}\text { Diabetes- } \\
\text { related ER } \\
\text { visits (\%) }\end{array}$ & $0.0 \%$ & $8.5 \%$ & $5.2 \%$ & $35.6 \%$ & $80.8 \%$ & $9.3 \%$ & $4.0 \%$ \\
\hline $\begin{array}{l}\text { Diabetes- } \\
\text { related IP visits } \\
(\%)\end{array}$ & $11.2 \%$ & $7.3 \%$ & $5.4 \%$ & $95.7 \%$ & $21.3 \%$ & $5.8 \%$ & $2.7 \%$ \\
\hline $\begin{array}{l}\text { Diabetes- } \\
\text { related IP days } \\
\text { for patients } \\
\text { with } \geq 1 \text { admit } \\
\text { (mean, SD) }\end{array}$ & $3.81(2.02)$ & $3.87(2.67)$ & $4.05(2.79)$ & 23.64 (22.99) & $4.07(2.71)$ & $3.52(2.84)$ & $4.88(4$ \\
\hline \multirow[t]{5}{*}{$\begin{array}{l}\text { Cluster } \\
\text { Characteristics }\end{array}$} & \multirow{5}{*}{$\begin{array}{l}\text { - No patients with } \\
\text { all-cause or } \\
\text { diabetes-related } \\
\text { ER visits } \\
\text { - Second highest } \\
\text { mean CCI and } \\
\text { DCSI scores }\end{array}$} & \multirow{5}{*}{$\begin{array}{l}\text { - } 100 \% \text { had an } \\
\text { Endocrinologist } \\
\text { visit } \\
\text { - Highest } \\
\text { proportion with } 1 \\
+ \text { HbA1c tests } \\
\text { performed }(76 \%)\end{array}$} & \multirow[t]{5}{*}{$\begin{array}{l}\text { - Highest average } \\
\text { HbA1c lab value } \\
\text { (mean = 9.35) }\end{array}$} & \multirow[t]{2}{*}{$\begin{array}{l}\text { - Highest } \\
\text { proportion of new } \\
\text { insulin users } \\
\text { (mean }=2.68 \\
\text { months) }\end{array}$} & \multirow{2}{*}{$\begin{array}{l}\cdot 100 \% \text { had an ER } \\
\text { visit } \\
\cdot 81 \% \text { had a } \\
\text { diabetes-related } \\
\text { ER visit }\end{array}$} & $\begin{array}{l}\text { - Highest } \\
\text { proportion of type } \\
1 \text { diabetics }(70 \%)\end{array}$ & \multirow{3}{*}{$\begin{array}{l}\text { - Healtl } \\
\text { accord } \\
\text { and DC } \\
\text { - Large } \\
\text { proport } \\
\text { withou } \\
\text { Endocr } \\
\text { or Car } \\
\text { visits }\end{array}$} \\
\hline & & & & & & $\begin{array}{l}\cdot \text { Youngest (mean } \\
\text { age }=38 \text { years) }\end{array}$ & \\
\hline & & & & $\begin{array}{l}\mathrm{CCl} \text { and DCSI } \\
\text { scores }\end{array}$ & \multirow[t]{3}{*}{$\begin{array}{l}\text { - Higher observed } \\
\text { HbA1c (mean = } \\
8.95)\end{array}$} & $\begin{array}{l}\text { - Relatively high } \\
\text { proportion with an } \\
\text { Endocrinology }\end{array}$ & \\
\hline & & & & $\begin{array}{l}\cdot \text { Highest average } \\
\text { number of } \\
\text { medical claims } \\
\text { (median }=43 \text { ) }\end{array}$ & & $\begin{array}{l}\text { visit (39\%) } \\
\text { - Highest diabetes } \\
\text { technology use } \\
\text { (insulin pump }\end{array}$ & \multirow[t]{2}{*}{$\begin{array}{l}- \text { Lowe } \\
\text { numbe } \\
\text { medicé } \\
\text { (media }\end{array}$} \\
\hline & & & & $\begin{array}{l}\text { - Nearly } 100 \% \text { had } \\
\text { an Inpatient } \\
\text { Hospitalization }\end{array}$ & & $\begin{array}{l}\text { CGM, and/or } \\
\text { BGM) }\end{array}$ & \\
\hline
\end{tabular}

Abbreviations: $\mathrm{BGM}=$ blood glucose monitor; $\mathrm{CCI}=$ Charlson Comorbidity Index; $\mathrm{CGM}=$ continuous glucose monitor; $\mathrm{DCSI}=$ diabetes complications severity ir emergency room; $\mathrm{HbA} 1 \mathrm{c}=$ hemoglobin $\mathrm{A} 1 \mathrm{c} ; \mathrm{IP}=$ inpatient; $\mathrm{n}, \mathrm{N}=$ number of people with diabetes; $\mathrm{PCP}=$ primary care physician; $\mathrm{Q} 1=$ first quartile; $\mathrm{Q} 3=$ third qi = standard deviation; T1DM = type 1 diabetes mellitus.

${ }^{1}$ Missing values for median household income were imputed for 454 patients for the purpose of cluster analysis.

2 Proportion is set to zero for those members who had zero medical claims overall during the baseline period.

${ }^{3}$ Diabetes related determined by diagnosis in any diagnostic position on a medical claim.

${ }^{4}$ Statistical comparisons for average utilization were conducted using Kruskal-Wallis tests.

The seven clusters fell into three main groupings in hierarchical order (Fig. 3): those with endocrinology visits (Clusters 2 and 6) with differing concordant burden profiles; those with high acute care utilization (Clusters 4 and 5) with differences in inpatient days and ER visits; and those with low ER utilization (Clusters 1,3 , and 7) with varying comorbidity burden, diabetes utilization, and complication profiles. 


\section{Endocrinology utilizers clusters}

Cluster 2 all had at least one endocrinologist visit (mean $=1.8$ visits/patient) and the average number of concordant comorbidities was 4.6 . Cluster 6 had the youngest mean age (38.3 years) and the second highest utilization of endocrinologists (mean $=0.6$ visits/patient) but had few concordant comorbidities (mean $=0.5$ ). Additionally, the DCSI and CCI scores were relatively low.

\section{High acute care utilizers clusters}

Those in Cluster 4 had the highest number of inpatient days with nearly all (99.1\%) visiting the hospital at least once with the vast majority having an inpatient stay that was diabetes related (95.7\%). Cluster 4 also had the highest overall number of medical claims and the highest comorbidity and complications burden (per the $\mathrm{CCl}$ and DCSI). Cluster 5 all experienced an ER visit, usually related to diabetes (80.8\%).

\section{Low ER utilizers clusters}

Those people in Clusters 1, 3, and 7 had few ER and endocrinology visits. Cluster 1 had a relatively high complication and comorbidity burden, second only to DCSI and CCI scores in Cluster 4, but those people in Cluster 1 had no ER utilization. In contrast, Clusters 3 and 7 had relatively low CCI and DCSI scores. Cluster 7 had the lowest overall number of medical claims (median $=3.00$ ) and the lowest CCl scores (mean $=0.30)$ suggesting that Cluster 7 was the healthiest population, however only $15.2 \%$ of those in Cluster 7 had at least one HbA1c test performed. While those in Cluster 7 had nearly no diabetes-related claims (mean $=0.19)$, more than three-quarters of the claims for Cluster 3 were diabetes related $(\operatorname{mean}=0.78)$.

Other notable differences were observed across clusters for variables not included in the cluster formations. Clusters 6 and 2 had the highest proportion of T1DM patients (69.6\% and $13.6 \%$, respectively) and diabetes technology use (pump, CGM, or BGM). Consistent with the young mean age, nearly all patients in Cluster 6 had commercial insurance. The highest proportion of Medicare Advantage members (and oldest mean age) was in Clusters 1 and 4 (> $80 \%$ ). The highest mean $\mathrm{HbA} 1 \mathrm{c}$ value (among the subset of the population with available values) was in Cluster 3 (9.4\%). On the other hand, the lowest mean $\mathrm{HbA} 1 \mathrm{c}$ of $8.3 \%$ was observed in Cluster 4 , which also had the highest proportion without prior insulin use in the pre-period and the lowest mean number of months where insulin was filled.

\section{Multivariable modeling analysis}

For the multivariable modeling analysis, three key outcomes were chosen including all-cause ER visit (Fig. 4), all-cause inpatient hospitalization (Fig. 5), and total inpatient days (Fig. 6) during the follow-up period. Across all models, clusters were independently associated with the outcomes of interest after controlling for covariates that were potentially related to cluster assignment and/or outcomes of interest. Clusters 1, 2, 4, and 5 had higher odds of an ER visit, whereas Cluster 6 had lower odds compared to Cluster 7. Clusters 2, 4, and 5 also had higher odds of having a hospital admission. If hospitalized, Cluster 4 had significantly increased total inpatient days compared to Cluster 7.

\section{Discussion And Conclusions}

The aim of this study was to determine how clusters or subgroups of insulin-treated people with diabetes, based upon healthcare resource utilization, select social demographic/clinical characteristics, and diabetes management parameters, are related to health outcomes including acute care (ER and hospital inpatient) visits and total inpatient days.

We did this study to help identify groups of patients that may be amenable to emerging diabetes management technologies. In this study, we identified seven clusters of insulin-treated people with diabetes, which have different patterns of healthcare utilization and diagnosed comorbidities in a large healthcare claims database. The most important factors in defining the clusters were the number of endocrinology visits, total inpatient days, concordant comorbidities, number of ER visits, comorbidity burden as measured by CCI and DCSI scores, and percentage of diabetes-related medical claims. Multivariable modeling showed that these clusters are significantly associated with ER visits, inpatient hospitalizations, and total inpatient days, suggesting that this approach may help identify patients at greater need for targeted disease management efforts at the population level. The clusters also offer providers clinically relevant information regarding treatment decisions for a patient population with diabetes.

Cluster analyses can reveal how variables, in our case administrative claims from people with diabetes, are related in complex datasets. The use of cluster analyses in healthcare decision making is still relatively uncommon but appears to be gaining acceptance $[17,18,19,20,21]$. Our work builds upon a few previously published cluster analyses in diabetes, which focused on readiness of CGM and other diabetesrelated devices, self-management patterns in a pediatric population, and factors influencing people with diabetes who have poorly controlled conditions $[22,23,24,25,26]$. These previous studies involved smaller numbers of participants from relatively homogeneous populations (e.g., T1DM registry) and/or more controlled conditions (e.g., clinical trial). In contrast, our study used a large healthcare claims database to evaluate whether routinely available data could identify relevant subgroups of insulin users.

Not only did clusters differ with respect to the specific variables used to form them (by design), but also on important other characteristics. Clusters 2 and 6 were formed primarily based on the use of endocrinologists. Not surprisingly, these were the clusters with the highest proportion of people with T1DM as well as utilizers of diabetes technology (pump, BGM, or CGM). Those in Cluster 2, however, had higher comorbidity burden and mean number of HbA1c tests than those in Cluster 6, but there was little difference in mean $\mathrm{HbA1}$ c values for these two clusters.

Two clusters were identified with high levels of acute care utilization. Those in Cluster 4 had the highest total inpatient days and everyone in Cluster 5 had an ER visit. These clusters differed, however, in their comorbidity burdens and glycemic control. Interestingly, the lowest mean observed $\mathrm{HbA} 1 \mathrm{c}$ value was for Cluster 4, with the highest levels of overall medical utilization (median of 43.0 claims), acute care utilization ( $99.1 \%$ had an inpatient hospitalization), and highest $\mathrm{CCl}$ and DCSI scores. These results could suggest that a high burden of comorbidities or diabetes complications and increased interactions with 
hospitals facilitated more intensive diabetes management. However, because $\mathrm{HbA} 1 \mathrm{c}$ values were only available on a subset of the study population (approximately $30 \%$ ), additional analyses on datasets with more complete HbA1c data are needed to confirm this finding.

Conversely, higher mean HbA1c's were observed among Clusters 3, 5, and 7 (in order of highest to lowest values).

Clusters 3 and 7 differ from Cluster 5 in that they fell into the low utilization grouping (both acute care and overall utilization via number of medical claims) and had among the lowest $\mathrm{CCl}$ and DCSI scores.

They differed from each other in one key aspect: the proportion of medical claims that were diabetes-related. Approximately three-fourths of the claims for Cluster 3 were related to diabetes, compared to less than $20 \%$ in Cluster 7 . Because the $\mathrm{CCI}$ and DCSI scores are derived from the presence of diagnosis codes in claims data, on one hand it is not surprising for these clusters who have the lowest overall number of medical claims to have the lowest scores due to fewer opportunities to derive those diagnoses. But, on the other hand, the lack of diagnoses of comorbidities in the observed claims or lack of encounters altogether could suggest a healthier underlying population. Either way, the relatively high observed $\mathrm{HbA} 1 \mathrm{c}$ values along with the low rates of interactions with healthcare providers suggested suboptimal diabetes self-management.

The current study demonstrated that even after adjusting for other covariates, cluster assignment was significantly predictive of future outcomes. Specifically, cluster assignment was associated with the likelihood of experiencing an ER or hospital inpatient visit and the total number of inpatient days for those with an admission. These results suggest that the specific combination of variables used in the cluster formations shed additional light onto the risk of untoward outcomes above and beyond traditional risk stratification, for example, based upon parameters including diabetes type, age, and HbA1c.

Furthermore, as these clusters were derived from variables routinely found in healthcare claims data where detailed clinical data are often missing, this approach could aid healthcare payers with population management efforts. We found some clusters utilizing less healthcare resources had higher observed mean $\mathrm{HbA} 1 \mathrm{c}$ levels. This finding could suggest population management efforts in diabetes that are targeted at some of the lower healthcare utilizers in efforts to improve glycemic control, which could yield better long-term health outcomes for patients and improved quality metric ratings for providers and payers.

This study has limitations that should be considered. The cluster analysis was based on realworld claims data that are proxies for clinical outcomes. Therefore, there may be data coding errors and errors in patient records because of the standardized coding systems used for the identification of medical conditions, procedures, and medications. Additionally, as of October 2015, all claims switched from ICD9 to ICD-10 (study period January 2015 to June 2018) [27]. This switch possibly could have led to inaccuracies in coding due to unfamiliarity with the new system or mistakes in cross walking codes from ICD-9 to ICD-10 by providers. This potential for error should have had limited impact since condition identification was based on families of codes with multiple codes within a family and not on any single code. The comparisons of HbA1c values were incomplete as only a subset of patients ( 30\%) had values in the database. A number of relevant risk factors, including insulin dosing, diet, and exercise, were not available in the database.

Despite the limitations, this study was based on a well-developed study design and included data from a large number of insulin-using people with either T1DM or T2DM. Evaluating the impact of patientreported outcomes and more socioeconomic data on cluster formations would be of interest to study in the future.

\section{Conclusion}

In conclusion, we demonstrated that clustering analyses of healthcare claims data identified meaningful subgroups of patients that differed in comorbidity burden, healthcare utilization, and demographic factors.

These clusters were found to be significantly associated with future outcomes indicating that providers and population health managers may be able to better estimate risk, based upon combinations of specific variables, and modify and/or personalize treatment accordingly.

\section{Abbreviations}

$\mathrm{BGM}=$ blood glucose monitor; $\mathrm{CART}$ = classification and regression trees; $\mathrm{CCl}=$ Charlson comorbidity index; $\mathrm{CGM}=$ continuous glucose monitoring; $\mathrm{DCSI}=$ diabetes complications severity index; $\mathrm{ER}=$ emergency room; HbA1C = hemoglobin A1C; ICD = International Classification of Diseases; IP = inpatient; $\mathrm{PCP}=$ primary care physician; $\mathrm{SD}=$ standard deviation; T1DM = type 1 diabetes mellitus; T2DM = type 2 diabetes mellitus; US, USA = United States/United States of America.

\section{Declarations}

\section{Ethics approval and consent to participate}

This study complies with relevant guidelines and regulations on observational studies. The study was approved by the Sterling Institutional Review Board (Atlanta, Georgia, USA). A waiver of informed consent was granted by the Sterling Institutional Review Board. All of the data used in this study were fully de-identified and Health Insurance Portability and Accountability Act compliant.

Consent for publication

Not applicable. 
The datasets generated and/or analyzed during the current study are not publicly available due to their proprietary nature, having been licensed only for the current study, and their protection under the Health Insurance Portability and Accountability Act (HIPAA). Subject to the data owner's determination of reasonableness and only after execution of a confidentiality agreement with the data owner, among other requirements, the data is available from the corresponding author upon reasonable request

\section{Competing interests}

ELE, EM, IL, and BDB are employees and shareholders of, and serve as authors for Eli Lilly and Company and AE and KS are employees of, and serve as authors for CVS Health Clinical Trial Services LLC.

Funding

This work was supported by Eli Lilly and Company.

Author contributions

ELE, EM, BDB, and KS conceptualized the study; AE and IL conducted the analyses; ELE and AE wrote the manuscript; and EM, BDB, KS, and IL critically commented on the manuscript. All authors read and approved the final manuscript.

\section{Acknowledgments}

The authors wish to acknowledge Debra Hidayetoglu for providing medical writing services on behalf of Covance, Inc.

Author information

Affiliations

Eli Lilly and Company, Lilly Corporate Center, Indianapolis, Indiana 46285, USA

Elizabeth L Eby, Eric Meadows, llya Lipkovich, and Brian D Benneyworth

CVS Health Clinical Trial Services LLC, 151 Farmington Avenue, Hartford, Connecticut 06156, USA

Alison Edwards and Kenneth Snow

\section{Corresponding author}

Correspondence to Elizabeth L Eby.

\section{References}

1. American Diabetes Association. Statistics about Diabetes. https://www.diabetes.org/resources/statistics/statistics-about-diabetes. Accessed 19 August 2019.

2. American Diabetes Association. 1. Introduction: Standard of Medical Care in Diabetes - 2019. Diabetes Care 2019;42(Suppl. 1):S1-S2 I https://doi.org/10.2337/dc19-SINT01. https://care.diabetesjournals.org/content/42/Supplement_1. Accessed 19 August 2019.

3. Centers for Disease Control and Prevention. National Diabetes Statistics Report, 2017. Atlanta, GA: Centers for Disease Control and Prevention, U.S. Dept of Health and Human Services; 2017.

4. American Diabetes Association. 2. Classification and Diagnosis of Diabetes: Standards of Medical Care in Diabetes - 2019. Diabetes Care 2019;42(Suppl. 1):S13-S28. https://doi/10.2337/dc19-S002.

5. American Diabetes Association. 9. Pharmacologic approaches to glycemic treatment: Standards of Medical Care in Diabetes - 2019. Diabetes Care. 2019;42(Suppl. 1):S90-S102. https://doi/10.2337/dc19-S009.

6. American Diabetes Association. Economic costs of diabetes in the U.S. in 2017. Diabetes Care. 2018;41:917-928. https://doi/10.2337/dci18-0007.

7. American Diabetes Association. 7. Diabetes Technology:Standards of Medical Care in Diabetes - 2019. Diabetes Care. 2019;42(Suppl. 1):S71-S80. https://doi/10.2337/dc19-S007.

8. Fry A. Insulin delivery device technology 2012: where are we after 90 years? J Diabetes Sci Technol. 2012;6(4):947-953. https://doi/10.1177/193229681200600428.

9. Perez-Nieves M, Jiang D, Eby E. Incidence, prevalence, and trend analysis of the use of insulin delivery systems in the United States (2005 to 2011$)$. Curr Med Res Opin. 2015;31:5, 891-899. https://doi/10.1185/03007995.2015.1020366.

10. Spollett GR. Improved disposable insulin pen devices provide an alternative to vials and syringes for insulin administration. Diabetes Spectrum. 2012; 25(2):117-122. https://doi/10.2337/diaspect.25.2.117.

11. Selvin E, Parrinello CM, Daya N, Bergenstal RM. Trends in insulin use and diabetes control in the U.S.: 1988-1994 and 1999-2012. Diabetes Care. 2016;39(3):e33-35. https://doi/10.2337/dc15-2229.

12. Hanchak NA, Murray JF, Hirsch A, McDermott PD, Schlackman N. USQA health profile database as a tool for health plan quality improvement. Manag Care Q. 1996;4:58-69. Available at: https://www.ncbi.nlm.nih.gov/pubmed/10157263. Accessed 15 October 2019.

Page $12 / 18$ 
13. Young BA, Lin E, Von Korff M, et al. Diabetes complications severity index and risk of mortality, hospitalization, and healthcare utilization. Am J Manag Care. 2008;14(1):15-23.

14. Charlson ME, Pompei P, Ales KL, MacKenzie CR. A new method of classifying prognostic comorbidity in longitudinal studies: development and validation. J Chronic Dis. 1987;40(5):373-383. https://doi/10.1016/0021-9681(87)90171-8.

15. Piette JD, Kerr EA The impact of comorbid chronic conditions on diabetes care. Diabetes Care. 2006 Mar;29(3):725-31. https://doi/10.2337/diacare.29.03.06.dc05-2078.

16. Sugar CS, James GM. Finding the number of clusters in a dataset. J Am Stat Assoc. 2003;98:750-763. https://doi/10.1198/016214503000000666.

17. Cornell JE, Pugh JA, Williams JW, et al. Multimorbidity clusters: clustering binary data from multimorbidity clusters: clustering binary data from a large administrative medical database. Appl Multivar Res. 2017:12 (3):163-182. https://doi//10.22329/amr.v12i3.658.

18. Dilts D1, Khamalah J, Plotkin A. Using cluster analysis for medical resource decision making. Med Decis Making. 1995:15(4):333-347. https://doi/10.1177/0272989X9501500404.

19. Newcomer SR, Steiner JF, Bayliss EA. Identifying subgroups of complex patients with cluster analysis. Am J Manag Care. 2011;17(8):e324-e332.

20. Liao M, Li Y, Kianifard F, et al. Cluster analysis and its application to healthcare claims data: a study of end-stage renal disease patients who initiated hemodialysis. BMC Nephrology. 2016:17:25. https://doi/10.1186/s12882-016-0238-2.

21. van den Berge, MJC, Free RH, Arnold R, et al. Cluster analysis to identify possible subgroups in tinnitus patients. Front Neurol. 2017:8:115. https://doi/10.3389/fneur.2017.00115.

22. Shahreza FA, Hazar N. Using routine data to categorize poor control diabetic patients: an application of cluster analysis technique. Iran J Public Health. 2017:46 (1):120-127.

23. Tanenbaum ML, Adams RN, Lanning MS, et al. Using cluster analysis to understand clinician readiness to promote continuous glucose monitoring adoption. J Diabetes Sci Technol. 2018:12(6):1108-1115. https://doi/10.1177/1932296818786486.

24. Tanenbaum ML, Adams RN, Lanning MS, et al. From Wary Wearers to d-Embracers: personas of readiness to use diabetes devices. J Diabetes Sci Technol. 2018:12(6):1101-1107. https://doi/10.1177/1932296818793756.

25. Ahlquist E, Storm P, Käräjämäki A, et al. Novel subgroups of adult-onset diabetes and their association with outcomes: a data-driven cluster analysis of six variables. Lancet Diabetes Endocrinol. 2018:6(5):361-369. https://doi/10.1016/S2213-8587(18)30051-2.

26. Rohan JM, Rohan JM, Delamater A, et al. Identification of self-management patterns in pediatric type 1 diabetes using cluster analysis. Pediatric Diabetes. 2011:12:611-618. https://doi/10.1111/j.1399-5448.2010.00752.x.

27. US Department of Health and Human Services: HIPAA administrative simplification: modifications to medical data code set standards to adopt ICD-10CM and ICD10-PCS. [FR Doc. E9-743.] Fed Reg 2014; 79(149): 45128. https://www.govinfo.gov/content/pkg/FR-2014-08-04/pdf/2014-18347.pdf.

\section{Figures}

100,650 individuals ( $12 \%$ Type 1 and $88 \%$ Type 2 ) were identified for analysis during study period*

Total individuals with at least 12 months

of medical and pharmacy coverage

Identified as diabetics in Aetna HPD ${ }^{\circledR}$

Type classification al gorithm

Identified as insulin users

After clinical exclusions*

Age $\geq 18$ years on Index date

6 months baseline and 12 months follow-up

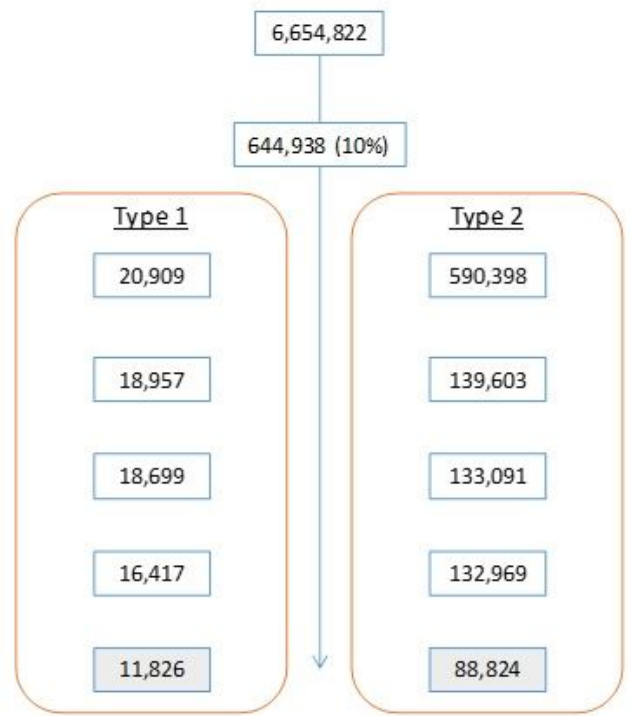

* Study period: January 1, 2015 through June 30, 2018

** Clinical exclusion: Gestational diabetes, Ste roid induced diabetes, Metastatic Cancer, Hospice or ACCP

Index date: Either the first insulin fill OR 6 months of e ligibility

Figure 1

Page 13/18 
Member selection - population funnel

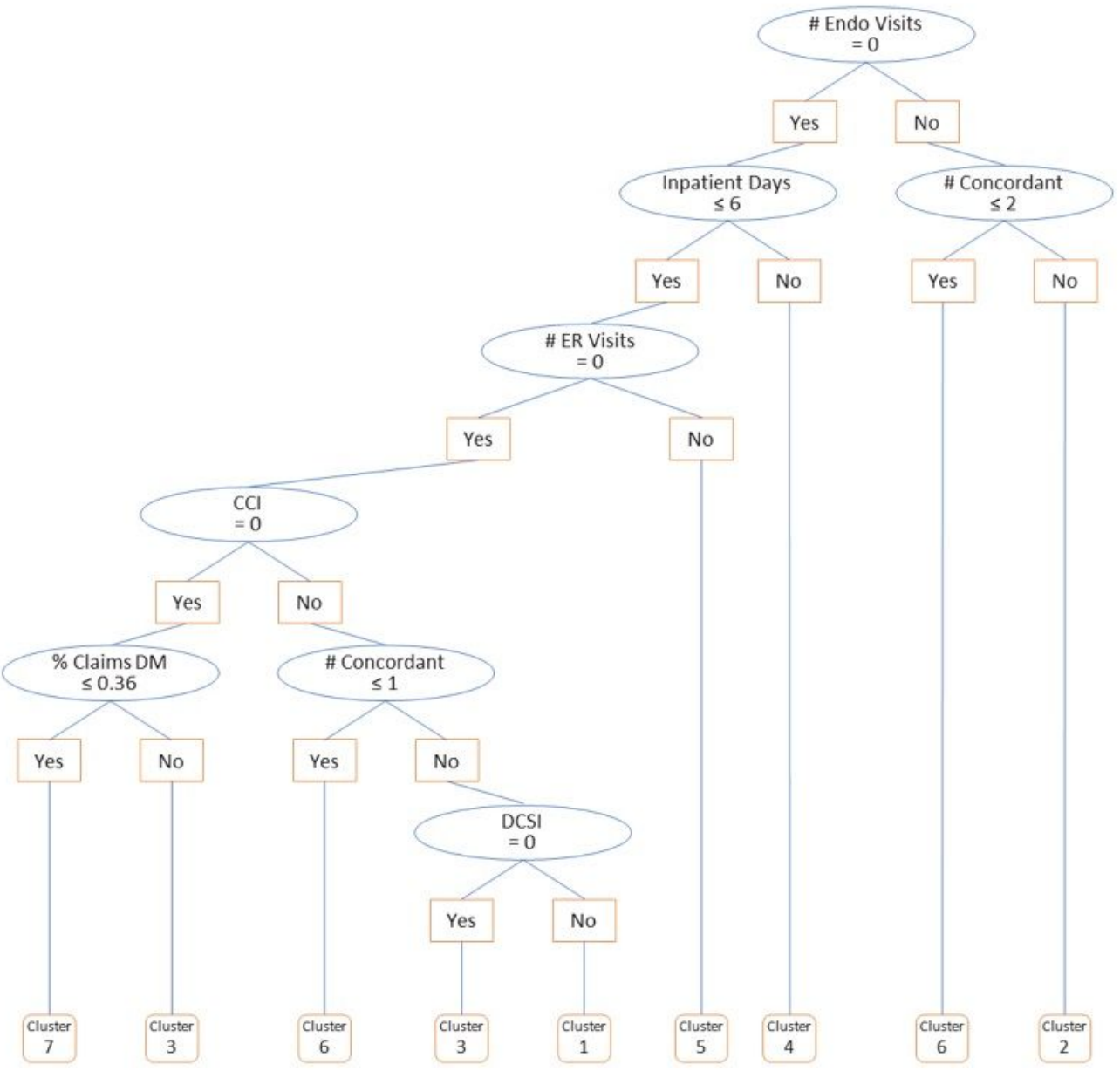

Note: Rules within the tree have been transformed back to original un-standardized definitions

Abbreviations: $\mathrm{CCI}=$ Charlson Comorbidity Index; DCSI $=$ Diabetes Complications Severity Index; $\mathrm{DM}=$ diabetes mellitus; Endo $=$ endocrinologist; $\mathrm{ER}=$ emergency room .

\section{Figure 2}

Cluster analysis schematic 

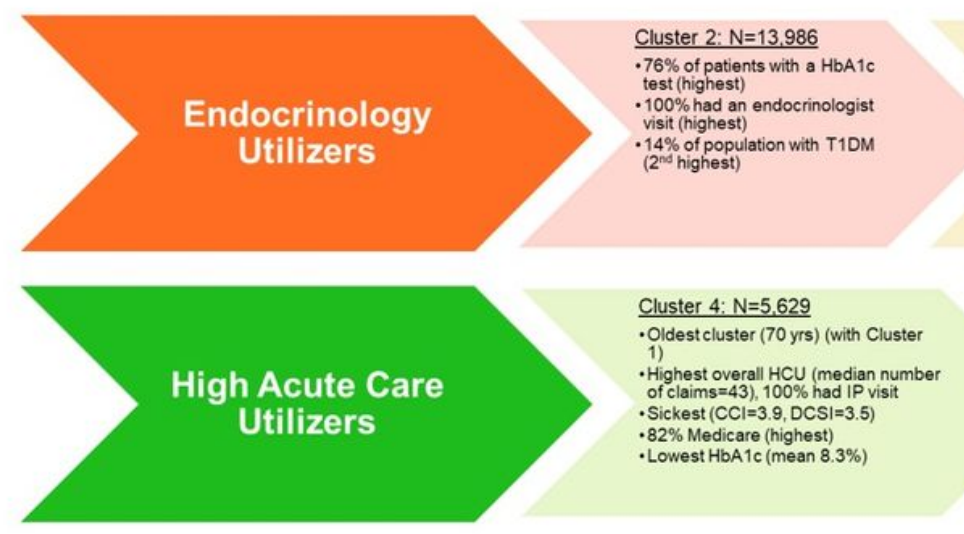

Cluster 4: $\mathrm{N}=5.62$

- Oldest cluster (70 yrs) (with Cluster

- Highest overall HCU (median number

-Sickest $(\mathrm{CCl}=3,9, \mathrm{DCSI}=3.5)$

Cluster 6: $N=10,049$

- $70 \%$ of population with T1DM

Younges

.96\% Commeup (38 yrs)

(highest)

- Highest tech use

- Low overall HCU (median

number of claims $=4$ )
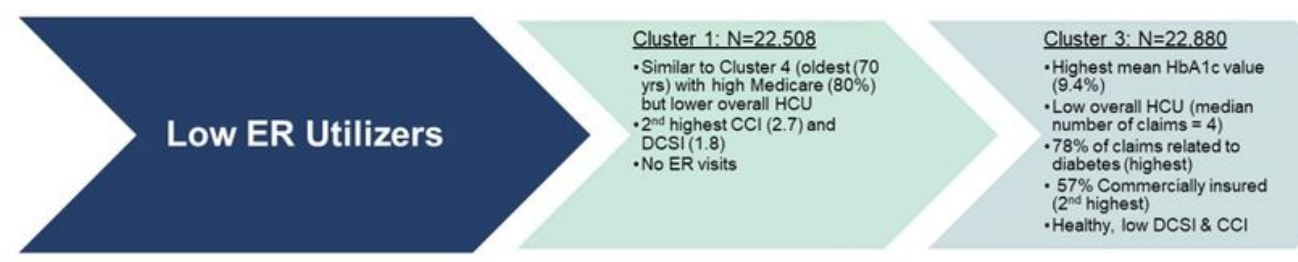

Cluster 5: $N=11,351$

- $100 \%$ had an ER visit

- $81 \%$ had a diabetes-related

- Higher mean $\mathrm{HbA} 1 \mathrm{C}=9.0 \%$

( $2^{\text {sad }}$ highest)

$\cdot 2^{\text {nd }}$ highest overall HCU

mber of claims $=14$ )

Abbreviations:

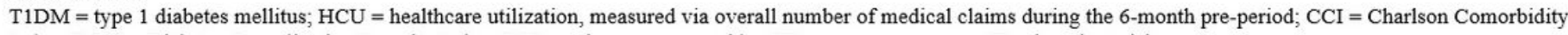
Index; DCSI = Diabetes Complication Severity Index; $\mathrm{PCP}=$ primary care provider; $\mathrm{ER}=$ emergency room; $\mathrm{IP}=$ inpatient visit

\section{Figure 3}

Cluster descriptions 


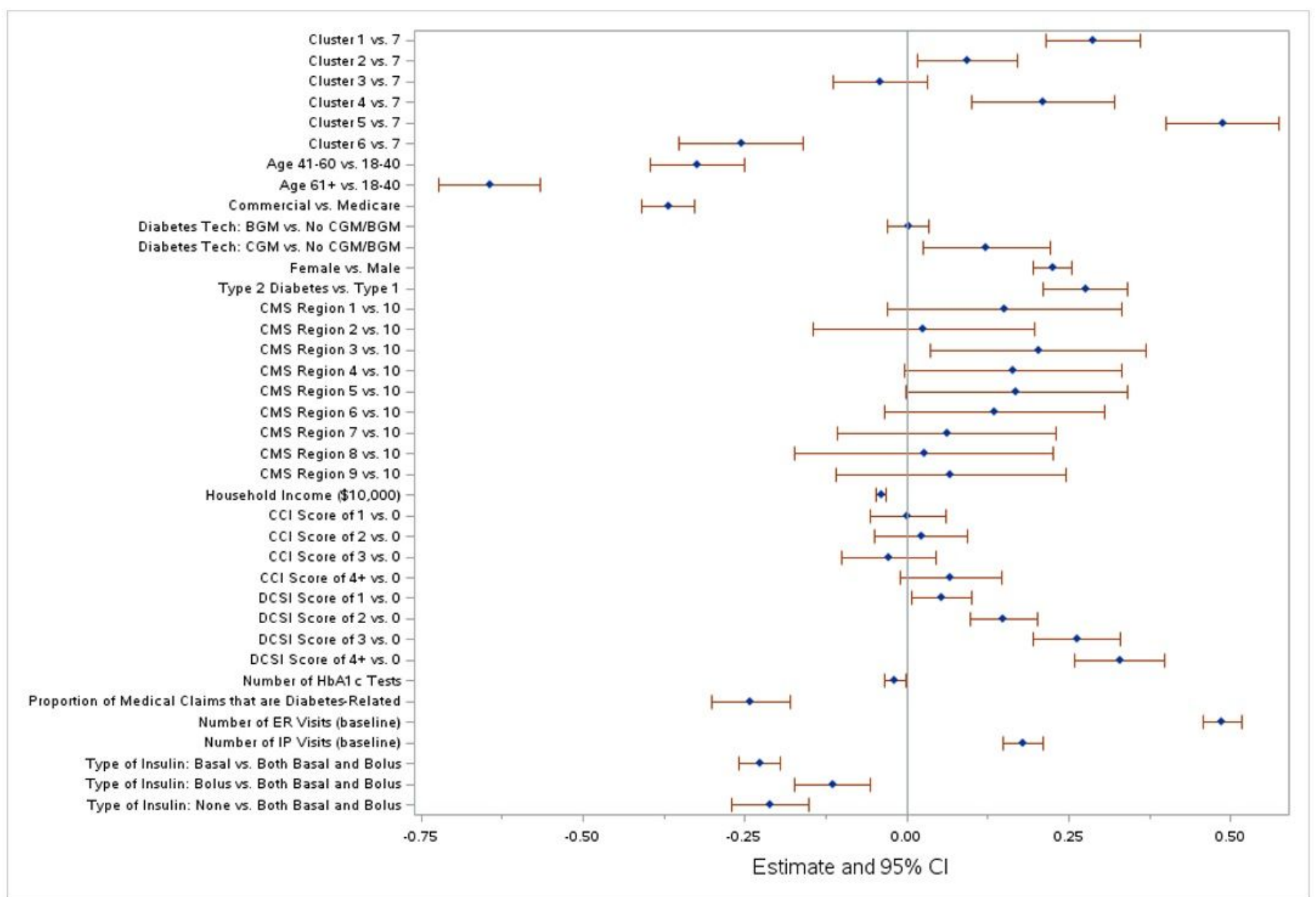

Abbreviations: $\mathrm{BGM}=$ blood glucose monitor; $\mathrm{CCI}=$ Charlson Comorbidity Index; $\mathrm{CGM}=$ continuous glucose monitor; $\mathrm{DCSI}=$ diabetes complications severity index; $\mathrm{ER}=$ emergency room; $\mathrm{HbA} 1 \mathrm{c}=$ hemoglobin $\mathrm{A} 1 \mathrm{c} ; \mathrm{IP}=$ inpatient .

\section{Figure 4}

All-cause emergency room visits - model results 


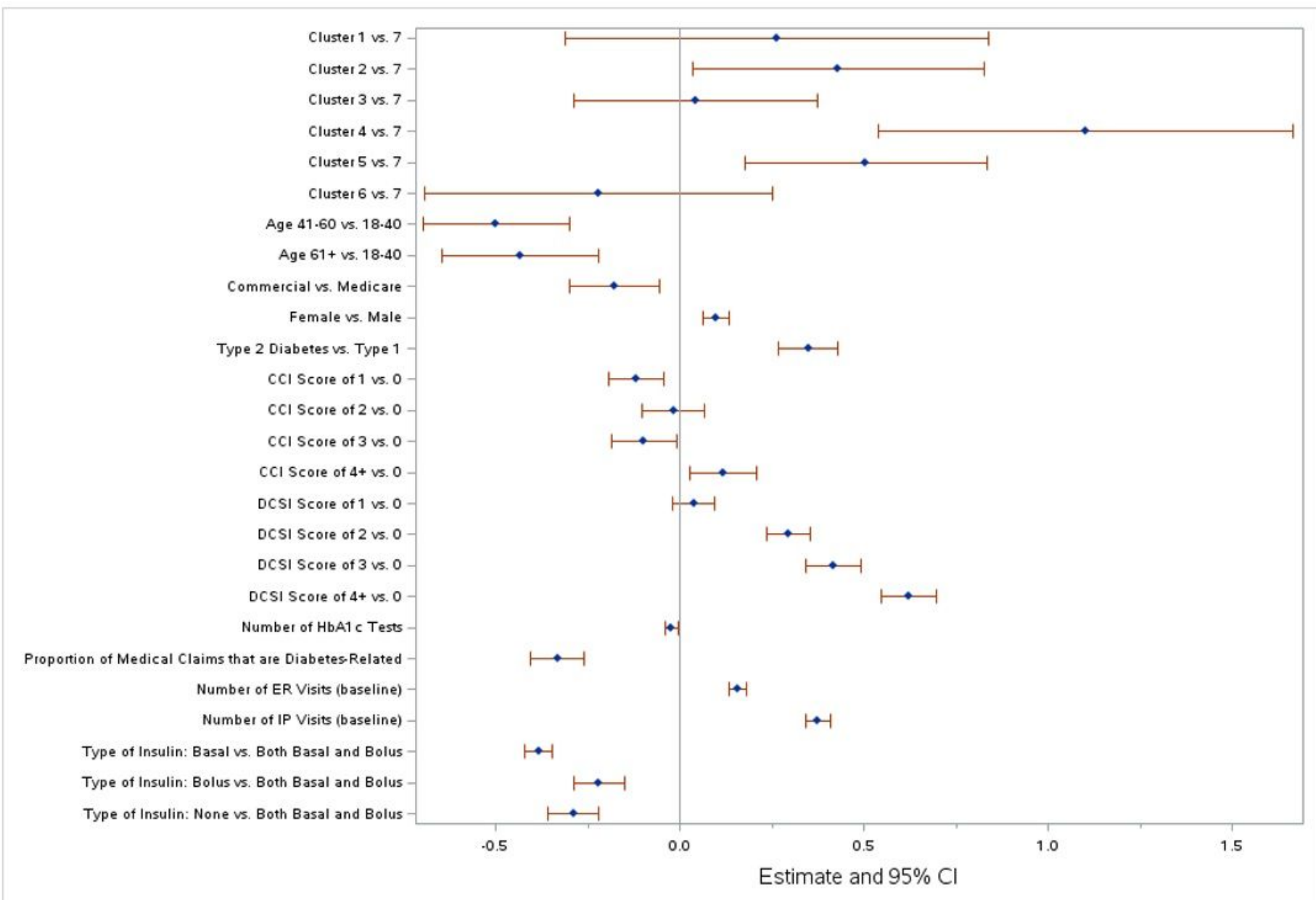

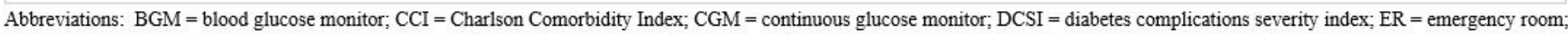
$\mathrm{HbA} 1 \mathrm{c}=$ hemoglobin $\mathrm{A} 1 \mathrm{c} ; \mathrm{IP}=$ inpatient.

\section{Figure 5}

All-cause inpatient hospitalization - model results 


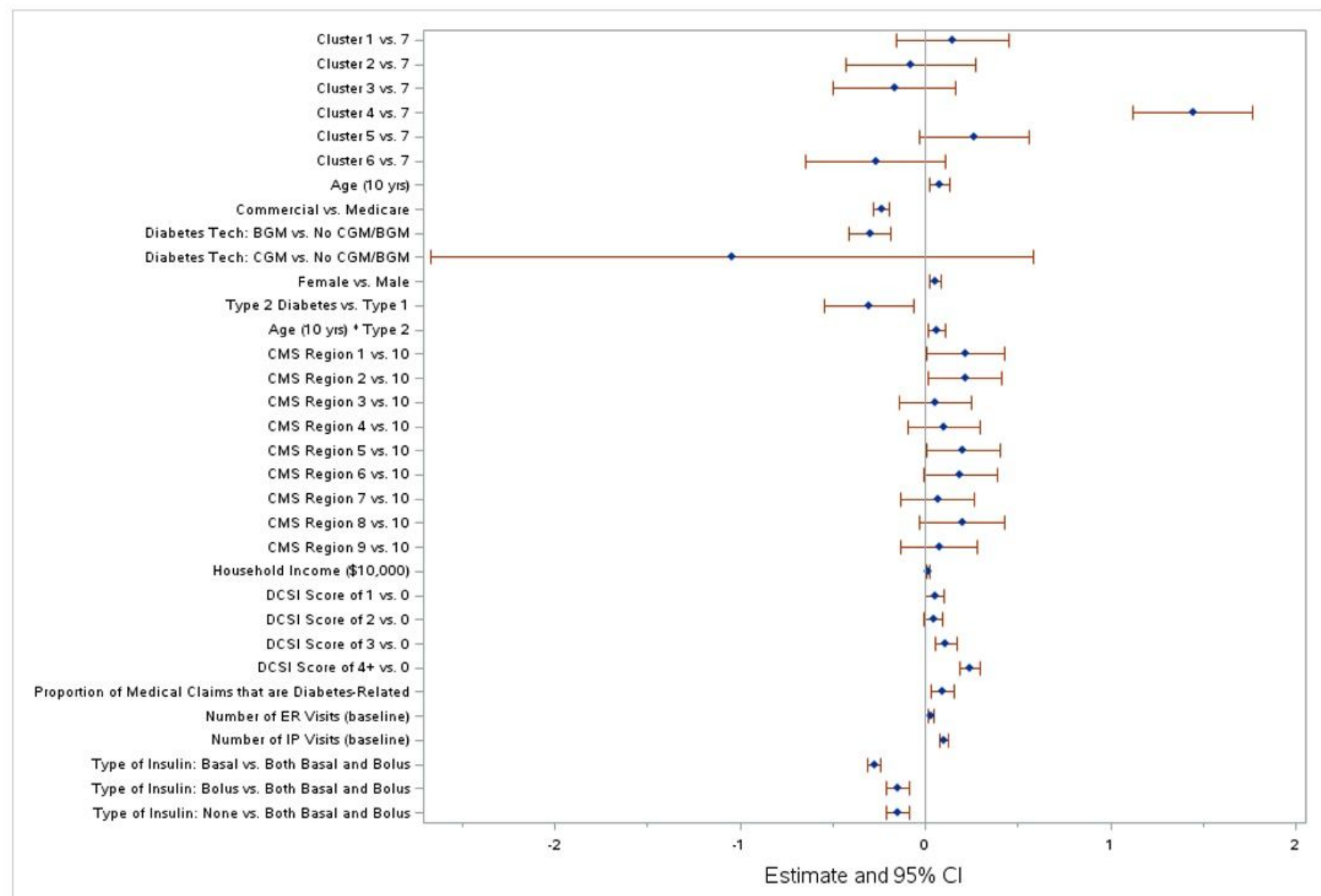

Abbreviations: $\mathrm{BGM}=$ blood glucose monitor; $\mathrm{CCI}=$ Charlson Comorbidity Index; $\mathrm{CGM}=$ continuous glucose monitor; $\mathrm{DCSI}=$ diabetes complications severity index; $\mathrm{ER}=$ emergency room; $\mathrm{HbA} 1 \mathrm{c}=$ hemoglobin $\mathrm{A} 1 \mathrm{c} ; \mathrm{IP}=$ inpatient.

\section{Figure 6}

Total inpatient days - model results

\section{Supplementary Files}

This is a list of supplementary files associated with this preprint. Click to download.

- SupplementalMaterials1TableS1forDiabetesClusterAnalysisManuscript05Feb2021.docx 